\title{
A BOUNDARY INTEGRAL METHOD FOR CONTAMINANT TRANSPORT IN TWO ADJACENT POROUS MEDIA
}

\author{
K. A. LANDMAN ${ }^{1}$ \\ (Received 24 August 1987; revised 19 January 1988)
}

\begin{abstract}
The problem of transient two-dimensional transport by diffusion and advection of a decaying contaminant in two adjacent porous media is solved using a boundaryintegral method. The method requires the construction of appropriate Green's functions. Application of Green's theorem in the plane then yields representations for the contaminant concentration in both regions in terms of an integral of the initial concentration over the region's interior and integrals along the boundaries of known quantities and the unknown interfacial flux between the two adjacent media. This flux is given by a first-kind integral equation, which can be solved numerically by a discretisation technique. Examples of contaminant transport in fractured porous media systems are presented.
\end{abstract}

\section{Introduction}

Contaminant transport by diffusion and advection in porous media has been a subject of intensive investigation recently. Much of this has been directed toward fractured porous media, since fractures constitute very effective pathways, the permeability and porosity of fractures being greater than the surrounding rock. It is a problem of great environmental relevance with obvious application to prediction of the consequences of leakage of radioactive or toxic waste from an underground repository.

Due to the complexities involved in attempting to solve the full transient two-dimensional problem in two adjacent media, analytical results can only be obtained by making simplifying assumptions. Specifically the transport in each medium is assumed to be mutually perpendicular. Then two one-dimensional

${ }^{1}$ Department of Mathematics, University of Melbourne, Parkville, Victoria 3052, Australia.

(C) Copyright Australian Mathematical Society 1989, Serial-fee code 0334-2700/89 
transport equations are obtained and they are coupled via a mass flux term across their interface $[6,8,10,12]$. These solutions are only good approximations within certain ranges of the parameters in the problems. A two-dimensional approach for steady state contaminant transport in fractured media using Fourier sine transforms has been developed which gives good analytical approximations [4].

Various numerical approaches to solution of the full problem have been proposed. Finite difference schemes have only proved useful when advection is a secondary transport mechanism, and are restricted by the use of rectangular elements. Finite element methods, based on the concept of weighted residuals, have shown more promise $[5,7,9]$. Many improvements and weighting schemes have been suggested to alleviate the difficulties of numerical oscillation, but these have the disadvantage of increasing both the complexity and the computational expense. In general there is still no convincing justification for the use of specific weights, apart from the fact that they give close agreement to analytical solutions for simple examples.

Another numerical approach is based on Green's theorem in the plane and the construction of an appropriate Green's function in each region where the unknown contaminant concentration is sought. Taigbenu and Liggett [11] consider a diffusion-advection transport equation in a single medium using this method. However, since they only employ the two-dimensional free-space Green's function solution of Laplace's equation, their expression for the contaminant concentration contains an integral over the region's interior (even when the initial concentration is zero), which must be evaluated using a finite element type technique.

In this paper we shall describe a new numerical method for solving the full two-dimensional transport problem in adjacent porous media. The governing equations are solved using a more complete Green's function treatment than that in Taigbenu and Liggett. Representations for the contaminant concentration in the interior of each medium are obtained in terms of an integral of the initial concentration over the region's interior, and boundary integrals of known quantities and the unknown interfacial flux between the two media. Applying the condition of continuity of the concentration across the interface gives a first-kind integral equation to be solved for this unknown flux. This method will be applied to examples commonly studied in fracture-matrix systems. The steady-state behaviour of such systems was discussed in a previous paper by Fogden, Landman and White [3], where a boundary-integral method was successfully implemented.

\section{Transport equation}

The general governing equation of two-dimensional contaminant transport in a saturated porous medium is, from Bear [2]: 


$$
R \partial c / \partial t+\nabla \cdot(\mathbf{V} c-\mathbf{D} \cdot \nabla c)+\lambda R c=0
$$

where $c$ is the contaminant concentration, $\mathbf{V}$ is the groundwater velocity vector $\left(V_{1}, V_{2}\right), \mathbf{D}$ is the hydrodynamic dispersion tensor, $\lambda$ is the decay constant and $R$ is the retardation coefficient due to adsorption of contaminant on the surfaces of the material making up the porous medium. The hydrodynamic dispersion tensor represents the process of dispersion due to fluid velocity variations and molecular diffusion. There is considerable discussion as to these coefficients' dependence on the flow field and media properties. With Bear's definition [2],

$$
D_{i j}=\left(\alpha_{T}|\mathbf{V}|+D^{*} \tau\right) \delta_{i j}+\left(\alpha_{L}-\alpha_{T}\right) \frac{V_{2} V_{j}}{|\mathrm{~V}|}, \quad i, j=1,2
$$

where $\alpha_{L}$ and $\alpha_{T}$ are the longitudinal and transverse dispersivities, $\tau$ is the tortuousity and $D^{*}$ is the molecular diffusion coefficient.

Typical boundary conditions for this equation are

(i) concentration $c$ specified on part of the boundary,

(ii) normal mass flux (per unit area of porous medium), $\theta(\mathbf{V} c-\mathbf{D} \cdot \nabla c) \cdot \mathbf{n}$, (where $\theta$ is the medium's porosity), specified on the remaining parts of the boundary, where $\mathbf{n}$ is the outward unit normal to the boundary. The initial concentration will also be known.

\section{Boundary integral formulation}

Introduce an operator $E$, defined in terms of an arbitrary function $u=u(x, y, t)$, by

$$
\begin{aligned}
E u= & -R \frac{\partial u}{\partial t}+\frac{\partial}{\partial x}\left(D_{11} \frac{\partial u}{\partial x}+D_{12} \frac{\partial u}{\partial y}-V_{1} u\right) \\
& +\frac{\partial}{\partial y}\left(D_{12} \frac{\partial u}{\partial x}+D_{22} \frac{\partial u}{\partial y}-V_{2} u\right)-\lambda R u .
\end{aligned}
$$

The operator $E^{*}$ defined by

$$
\begin{aligned}
E^{*} u= & R \frac{\partial u}{\partial t}+\frac{\partial}{\partial x}\left(D_{11} \frac{\partial u}{\partial x}+D_{12} \frac{\partial u}{\partial y}+V_{1} u\right) \\
& +\frac{\partial}{\partial y}\left(D_{12} \frac{\partial u}{\partial x}+D_{22} \frac{\partial u}{\partial y}+V_{2} u\right) \\
& -\left(\frac{\partial V_{1}}{\partial x}+\frac{\partial V_{2}}{\partial y}-\frac{\partial R}{\partial t}+\lambda R\right) u
\end{aligned}
$$


is the adjoint of $E$ since

$$
\begin{aligned}
u E^{*} w-w E u & =\frac{\partial}{\partial x}\left(u\left(D_{11} \frac{\partial w}{\partial x}+D_{12} \frac{\partial w}{\partial y}\right)-w\left(D_{11} \frac{\partial u}{\partial x}+D_{12} \frac{\partial u}{\partial y}-V_{1} u\right)\right) \\
& +\frac{\partial}{\partial y}\left(u\left(D_{12} \frac{\partial w}{\partial x}+D_{22} \frac{\partial w}{\partial y}\right)-w\left(D_{12} \frac{\partial u}{\partial x}+D_{22} \frac{\partial u}{\partial y}-V_{2} u\right)\right) \\
& +\frac{\partial}{\partial t}(R u w) .
\end{aligned}
$$

It is convenient now to define a mass flux vector (per unit area of voids)

$$
\mathbf{Q}(u)=\mathbf{V} u-\mathbf{D} \cdot \nabla u \text {. }
$$

Then (5) can be written in a simpler form as

$$
u E^{*} w-w E u=\frac{\partial}{\partial t}(R u w)+\nabla \cdot(\mathbf{V} u w-u \mathbf{Q}(w)+w \mathbf{Q}(u)) .
$$

If we replace $x, y, t$ in (7) by the integration variables $x^{\prime}, y^{\prime}, t^{\prime}$, integrate the equation over an arbitrary region $\Omega$ in the plane, bounded by the closed curve $\Gamma$, and integrate over $0 \leq t^{\prime} \leq T$ for a $T>t$, then by Green's theorem in the plane, we obtain

$$
\begin{aligned}
\int_{0}^{T} \iint_{\Omega}\left(u E^{*} w-w E u\right) d x^{\prime} d y^{\prime} d t^{\prime}= & \int_{0}^{T} \oint_{\Gamma}(\mathbf{V} u w-u \mathbf{Q}(w)+w \mathbf{Q}(u)) \cdot \mathbf{n} d s d t^{\prime} \\
& +\iint_{\Omega}\left((R u w)_{t^{\prime}=T}-(R u w)_{t^{\prime}=0}\right) d x^{\prime} d y^{\prime}
\end{aligned}
$$

where $\mathbf{n}$ is the outward unit normal to the boundary and $d s$ is an element of boundary arc length.

Now choose the function $u$ to be the contaminant concentration $c$, so that from (1) and the symmetry of $\mathbf{D}$ (from (2)), $E c=0$, and choose the function $w$ to be the Green's function $G$ satisfying the following partial differential equation (with respect to the primed variables).

$$
E^{*} G\left(x, y, t \mid x^{\prime}, y^{\prime}, t^{\prime}\right)=\delta\left(x^{\prime}-x\right) \delta\left(y^{\prime}-y\right) \delta\left(t^{\prime}-t\right)
$$

where the right-hand side is a product of delta functions. Then (8) simplifies to

$$
\begin{aligned}
c(x, y, t)= & \int_{0}^{T} \oint_{\Gamma}(\mathbf{V} c G-c \mathbf{Q}(G)+G \mathbf{Q}(c)) \cdot \mathbf{n} d s d t^{\prime} \\
& -\iint_{\Omega}(R c G)_{t^{\prime}=0} d x^{\prime} d y^{\prime}+\iint_{\Omega}(R c G)_{t^{\prime}=\boldsymbol{T}} d x^{\prime} d y^{\prime} .
\end{aligned}
$$

To solve the initial-value problem it is necessary for there to be no contribution to $c(x, y, t)$ for values of $t^{\prime}>t$; hence we set

$$
G=0, \quad t^{\prime}>t .
$$


Therefore the last integral in (10) vanishes, and the upper limit of integration in the first integral can be replaced by $t$ :

$$
c(x, y, t)=\int_{0}^{t} \oint_{\Gamma}(\mathbf{V} c G-c \mathbf{Q}(G)+G \mathbf{Q}(c)) \cdot \mathbf{n} d s d t^{\prime}-\iint_{\Omega}(R c G)_{t^{\prime}=0} d x^{\prime} d y^{\prime}
$$

So we have a representation for the contaminant concentration in the interior region of a porous medium in terms of (i) integrals along the region's boundaries and their value at all previous times and (ii) an integral of the initial concentration over the region's interior. For this representation to be useful, appropriate boundary conditions must be imposed on the Green's function. Progress has then been made provided that the solution of (9), subject to (11) and these boundary conditions, is a simpler task than solution of the original problem.

For those parts of the boundary $\Gamma$ where the concentration $c$ is prescribed, it is appropriate to choose $G=0$, while over the remaining parts of $\Gamma$ where the normal mass flux $\theta \mathbf{Q}(c) \cdot \mathbf{n}$ is specified, the boundary condition on $G$ should be $(\mathbf{V} G-\mathbf{Q}(G)) \cdot \mathbf{n}=(\mathbf{D} \cdot \nabla G) \cdot \mathbf{n}=\mathbf{0}$. Then the representation in (12) involves integrals along the boundaries of known quantities (concentration or mass flux) at previous times and an integral of the given initial concentration.

\section{Two medium problem}

In many physical situations, contaminant can be transported into two adjacent porous media, each with its own characteristic parameters $(\mathbf{D}, \mathrm{V}, R, \theta)$. One way to treat such a problem is to consider all the parameters as functions of position $(x, y)$. In general the Green's function solution cannot be obtained analytically so that the boundary integral representation in (12) is not useful. However if each medium has constant (but different) parameter values another approach is available. This involves applying the above boundary integral solution technique to each of the media separately. Then in each medium the concentration has a representation which involves quantities on the boundaries of the region. One of these boundaries is the interface between the two media, across which the contaminant concentration and normal mass flux must be continuous. Hence if the Green's function in each region is chosen to satisfy $(\mathrm{V} G-\mathbf{Q}(G)) \cdot \mathbf{n}=$ $(\mathrm{D} \cdot \nabla G) \cdot \mathbf{n}=\mathbf{0}$ along the interface, the concentrations in both regions have representations in terms of integrals along their boundaries of known quantities and unknown interfacial normal mass flux. This latter term in each medium has the form

$$
\int_{0}^{t} \int_{\substack{\text { interface } \\ \text { boundary }}} G \mathbf{Q}(c) \cdot \mathbf{n} d s d t^{\prime}
$$

where $\theta \mathbf{Q}(c) \cdot \mathbf{n}$ is the unknown interfacial flux (the Green's function will be different for each region). The continuity of concentration at the interface gives 
an equation for this interfacial flux in the following way. Evaluate the integral representations for $c$ in each medium along the interface boundary (it can be shown that each integral representation is valid not only in the interior of the medium but also along the interfacial boundary since the Green's function effectively satisfies a zero flux Neumann condition there). By equating these two expressions, an integral equation of the first kind for the interfacial mass flux is obtained. When this integral equation is solved (numerically) then the concentration everywhere in the two medium system at all times can be obtained.

In the next section this technique is applied to contaminant transport in a two medium system of wide interest, namely fractured porous media, which consists of fractures surrounded by a porous rock matrix. In Section 7 we discuss the integral equation for the interfacial flux which is applicable to any two medium problem.

\section{Fracture-matrix systems}

The occurrence of fractures in porous media is an important factor in the movement of a contaminant by diffusion and advection. They constitute very effective pathways, since the permeability and porosity of fractures are greater than those of the surrounding rock or soil.

A typical scenario in contaminant transport concerns a contaminant source in the vicinity of a fracture network leading to a freshwater aquifier. Since such networks may feasibly be of extremely intricate connected or disconnected form, attention is restricted to mathematically tractable "worst case" situations of uniform vertical fractures of constant width in uniform saturated porous media. In this section, the groundwater velocity is assumed to be vertical and constant in the fracture and the porous rock matrix. Also, it is assumed that in both regions contaminant diffusion occurs in both the vertical and horizontal directions. Further, absorption onto the matrix face and within the matrix and decay of the contaminant are all incorporated into this model.

Then the transport equation (1) in a single medium reduces to

$$
R \frac{\partial c}{\partial t}-D_{11} \frac{\partial^{2} c}{\partial x^{2}}-D_{22} \frac{\partial^{2} c}{\partial y^{2}}+V \frac{\partial c}{\partial y}+\lambda R c=0
$$

when $\mathrm{V}=\left(V_{1}, V_{2}\right)=(0, V)$ and all the transport coefficients and physical properties of the medium are constant. The boundary integral representation (12) 
becomes

$$
\begin{aligned}
c(x, y, t)= & \int_{0}^{t} \oint_{\Gamma} D_{11}\left(c \frac{\partial G}{\partial x^{\prime}}-G \frac{\partial c}{\partial x^{\prime}}\right) d y^{\prime} d t^{\prime} \\
& -\int_{0}^{t} \oint_{\Gamma}\left(D_{22}\left(c \frac{\partial G}{\partial y^{\prime}}-G \frac{\partial c}{\partial y^{\prime}}\right)+V c G\right) d x^{\prime} d t^{\prime} \\
& -\iint_{\Omega} R(c G)_{t^{\prime}=0} d x^{\prime} d y^{\prime}
\end{aligned}
$$

where the Green's function satisfies (9) and (11) with the adjoint operator $E^{*}$ defined by

$$
E^{*} u=R \frac{\partial u}{\partial t}+D_{11} \frac{\partial^{2} u}{\partial x^{2}}+D_{22} \frac{\partial^{2} u}{\partial y^{2}}+V \frac{\partial u}{\partial y}-\lambda R u
$$

Equations (13), (14) and (15) are valid for both the fracture and matrix regions, but the values of $R, D_{11}, D_{22}$ and $V$ are particular to each region. We shall use superscripts $f$ and $m$ on these parameters, to signify their value in the fracture and matrix respectively. We will analyse the transient contaminant transport for two "worst case" fracture-matrix systems. The steady state problems are considered in $[3,4]$. In both cases we let $b$ denote the half fracture width.

The first case is that of a porous medium, modelled by a half-plane, containing a single vertical fracture of infinite length, as shown in Figure 1(a). As in the previous paper [3], "worst case" considerations lead to the assumption that the boundary condition at $y=0$ is symmetric in $x$. Hence this symmetry is passed on to the entire problem and we need only solve the fracture transport equation in the region $0 \leq x<b, y>0$ and the matrix transport equation in the region $x>b, y>0$, imposing the boundary conditions

$$
\begin{gathered}
c(x, 0, t)=S(x, t), \quad x \geq 0, t>0, \\
\frac{\partial c}{\partial x}(0, y, t)=0, \quad y>0, t>0, \\
\frac{\partial c}{\partial x}(x, y, t) \rightarrow 0, \quad x \rightarrow \infty, y>0, t>0 \\
c(x, y, t) \rightarrow 0, \quad y \rightarrow \infty, x \geq 0, t>0
\end{gathered}
$$

and initial condition

$$
c(x, y, 0)=c_{0}(x, y) \quad x \geq 0, y>0 .
$$

Here the arbitrary functions $S(x, t)$ and $c_{0}(x, y)$ represent the source distribution of the contaminant at the top of the fracture-matrix system and the initial contaminant distribution in the system respectively.

The second case is that of a porous medium, again modelled by a half-plane, containing an infinite set of identical vertical fractures, of infinite length, with axes at equal spacing. This geometry is illustrated in Figure $1(\mathrm{~b})$, where $B$ is 


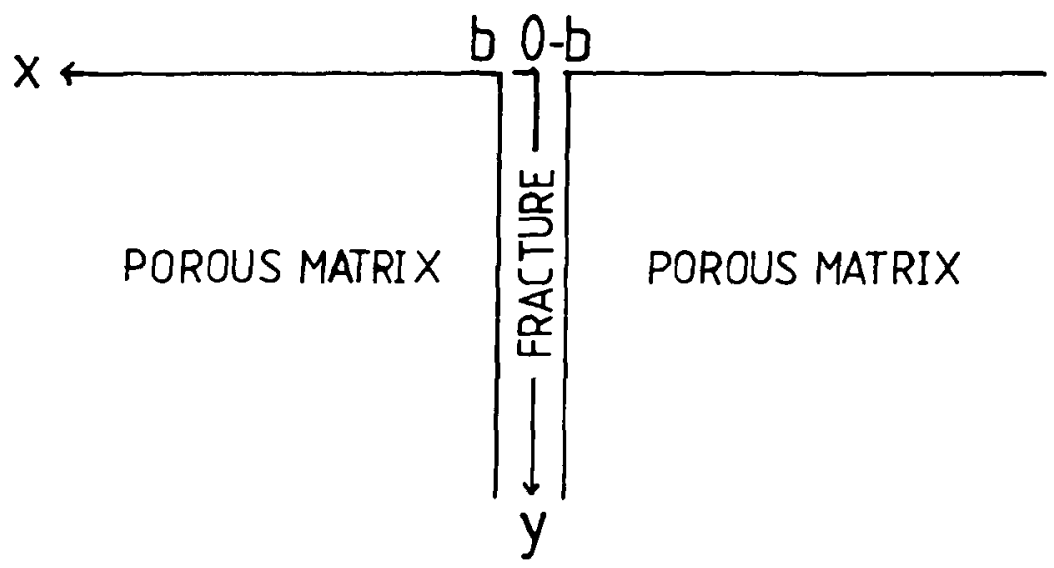

(a)

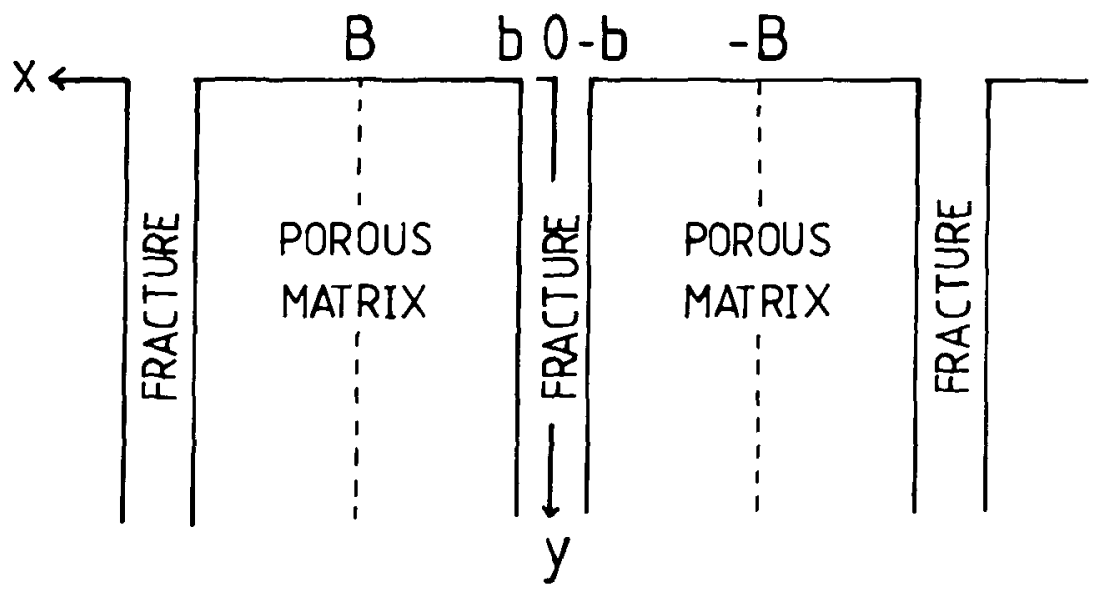

(b)

FiguRE 1. Configuration of the fracture-matrix system. (a) Single fracture problem (b) Periodic array of fractures problem.

the half fracture spacing. We assume that the boundary condition at $y=0$ is symmetric about the axis of symmetry of each fracture and each intervening porous block. Then the entire problem possesses these symmetries and we need only solve the fracture and matrix transport equations in the regions $0 \leq x<b$, $y>0$ and $b<x \leq B, y>0$ respectively. The appropriate boundary conditions for this geometry are then

$$
c(x, 0, t)=S(x, t) . \quad x \geq 0, t>0,
$$




$$
\begin{gathered}
\frac{\partial c}{\partial x}(0, y, t)=0, \quad y>0, t>0, \\
\frac{\partial c}{\partial x}(B, y, t)=0, \quad y>0, t>0, \\
c(x, y, t) \rightarrow 0, \quad y \rightarrow \infty, 0 \leq x \leq B, t>0
\end{gathered}
$$

and the initial condition is

$$
c(x, y, 0)=c_{0}(x, y), \quad 0 \leq x \leq B, y>0 .
$$

Note that the single fracture problem can be viewed as a special case of this periodic array of fractures problem in which the half fracture spacing $B$ is infinite.

Finally, for both geometries the problems in the fracture and matrix regions are coupled via the continuity of concentration and mass flux at the fracturematrix interface. This yields the two conditions

$$
\begin{aligned}
c\left(b^{+}, y, t\right) & =c\left(b^{-}, y, t\right) \\
\theta^{m} D_{11}^{m} \frac{\partial c}{\partial x}\left(b^{+}, y, t\right) & =\theta^{f} D_{11}^{f} \frac{\partial c}{\partial x}\left(b^{-}, y, t\right)
\end{aligned}
$$

where $\theta^{m}$ represents the matrix porosity, while the fracture porosity $\theta^{f}$ is usually assumed to be unity.

We now apply the boundary integral solutions (14) to the fracture and matrix regions separately for each of the two geometries considered. For both the single fracture and periodic array of fractures problems, the fracture region is $0 \leq x<b, y>0$. We choose the Green's function $G=G^{f}$ in this region to be the same for both cases. It is the solution of (9) with $E^{*}$ given in (15) (with an $f$ superscript added to $R, D_{11}, D_{22}$, and $V$ in the partial differential equation) subject to

$$
\begin{gathered}
G^{f}\left(x, y, t \mid x^{\prime}, 0, t^{\prime}\right)=0, \quad 0 \leq x^{\prime}<b, t^{\prime}>0, \\
\frac{\partial G^{f}}{\partial x^{\prime}}\left(x, y, t \mid 0, y^{\prime}, t^{\prime}\right)=0, \quad y^{\prime}>0, t^{\prime}>0, \\
\frac{\partial G^{f}}{\partial x^{\prime}}\left(x, y, t \mid b^{-}, y^{\prime}, t^{\prime}\right)=0, \quad y^{\prime}>0, t^{\prime}>0, \\
G^{f}\left(x, y, t \mid x^{\prime}, y^{\prime}, t^{\prime}\right) \rightarrow 0, \quad y^{\prime} \rightarrow \infty, 0 \leq x^{\prime}<b, t^{\prime}>0, \\
G^{f}\left(x, y, t \mid x^{\prime}, y^{\prime}, t^{\prime}\right)=0, \quad t^{\prime}>t, 0 \leq x^{\prime}<b, y^{\prime}>0 .
\end{gathered}
$$

The solution is given in the next section. Then, from (14), we have the following representation for the concentration in the fracture region:

$$
c(x, y, t)=-D_{11}^{f} \int_{0}^{t} \int_{0}^{\infty} G^{f}\left(x, y, t \mid b^{-}, y^{\prime}, t^{\prime}\right) \frac{\partial c}{\partial x^{\prime}}\left(b^{-}, y^{\prime}, t^{\prime}\right) d y^{\prime} d t^{\prime}+I^{f}(x, y, t)
$$


where the known function $I^{f}$ is the contribution from the integration along the top of the fracture and the integration of the initial distribution of the contaminant in the region:

$$
\begin{aligned}
I^{f}(x, y, t)= & -D_{22}^{f} \int_{0}^{t} \int_{0}^{b} S\left(x^{\prime}, t^{\prime}\right) \frac{\partial G^{f}}{\partial y^{\prime}}\left(x, y, t \mid x^{\prime}, 0, t^{\prime}\right) d x^{\prime} d t^{\prime} \\
& -R^{f} \int_{0}^{\infty} \int_{0}^{b} c_{0}\left(x^{\prime}, y^{\prime}\right) G^{f}\left(x, y, t \mid x^{\prime}, y^{\prime}, 0\right) d x^{\prime} d y^{\prime} .
\end{aligned}
$$

The matrix region for the single fracture and periodic array of fractures problems are $x>b, y>0$ and $b<x \leq B, y>0$ respectively. So the Green's function $G=G^{m}$, which is the solution of (9), using (15), (now with the addition of a $m$ superscript to $R, D_{11}, D_{22}$, and $V$ in the partial differential equation) in this region will clearly be different for each case. For the single fracture problem we impose the conditions:

$$
\begin{gathered}
G^{m}\left(x, y, t \mid x^{\prime}, 0, t^{\prime}\right)=0, \quad x^{\prime}>b, t^{\prime}>0, \\
\frac{\partial G^{m}}{\partial x^{\prime}}\left(x, y, t \mid b^{+}, y^{\prime}, t^{\prime}\right)=0, \quad y^{\prime}>0, t^{\prime}>0, \\
\frac{\partial G^{m}}{\partial x^{\prime}}\left(x, y, t \mid x^{\prime}, y^{\prime}, t^{\prime}\right) \rightarrow 0, \quad x^{\prime} \rightarrow \infty, y^{\prime}>0, t^{\prime}>0, \\
G^{m}\left(x, y, t \mid x^{\prime}, y^{\prime}, t^{\prime}\right) \rightarrow 0, \quad y^{\prime} \rightarrow \infty, x^{\prime}>b, t^{\prime}>0, \\
G^{m}\left(x, y, t \mid x^{\prime}, y^{\prime}, t^{\prime}\right)=0, \quad t^{\prime}>t, x^{\prime}>b, y^{\prime}>0,
\end{gathered}
$$

while for the periodic array of fractures problem we choose

$$
\begin{gathered}
G^{m}\left(x, y, t \mid x^{\prime}, 0, t^{\prime}\right)=0, \quad b<x^{\prime} \leq B, t^{\prime}>0, \\
\frac{\partial G^{m}}{\partial x^{\prime}}\left(x, y, t \mid b^{+}, y^{\prime}, t^{\prime}\right)=0, \quad y^{\prime}>0, t^{\prime}>0, \\
\frac{\partial G^{m}}{\partial x^{\prime}}\left(x, y, t \mid B, y^{\prime}, t^{\prime}\right)=0, \quad y^{\prime}>0, t^{\prime}>0, \\
G^{m}\left(x, y, t \mid x^{\prime}, y^{\prime}, t^{\prime}\right) \rightarrow 0, \quad y^{\prime} \rightarrow \infty, b<x^{\prime} \leq B, t^{\prime}>0, \\
G^{m}\left(x, y, t \mid x^{\prime}, y^{\prime}, t^{\prime}\right)=0, \quad t^{\prime}>t, b<x^{\prime} \leq B, y^{\prime}>0 .
\end{gathered}
$$

The solution to these problems is given in the next section. In both cases the representation for the concentration in the matrix region can be expressed as:

$$
c(x, y, t)=D_{11}^{m} \int_{0}^{t} \int_{0}^{\infty} G^{m}\left(x, y, t \mid b^{+}, y^{\prime}, t^{\prime}\right) \frac{\partial c}{\partial x^{\prime}}\left(b^{+}, y^{\prime}, t^{\prime}\right) d y^{\prime} d t^{\prime}+I^{m}(x, y, t)
$$

where again $I^{m}$ is a known function. It is given by

$$
\begin{aligned}
I^{m}(x, y, t)= & -D_{22}^{m} \int_{0}^{t} \int_{b}^{\infty} S\left(x^{\prime}, t^{\prime}\right) \frac{\partial G^{m}}{\partial y^{\prime}}\left(x, y, t \mid x^{\prime}, 0, t^{\prime}\right) d x^{\prime} d t^{\prime} \\
& -R^{m} \int_{0}^{\infty} \int_{b}^{\infty} c_{0}\left(x^{\prime}, y^{\prime}\right) G^{m}\left(x, y, t \mid x^{\prime}, y^{\prime}, 0\right) d x^{\prime} d y^{\prime}
\end{aligned}
$$


for the single fracture problem and

$$
\begin{aligned}
I^{m}(x, y, t)= & -D_{22}^{m} \int_{0}^{t} \int_{b}^{B} S\left(x^{\prime}, t^{\prime}\right) \frac{\partial G^{m}}{\partial y^{\prime}}\left(x, y, t \mid x^{\prime}, 0, t^{\prime}\right) d x^{\prime} d t^{\prime} \\
& -R^{m} \int_{0}^{\infty} \int_{b}^{B} c_{0}\left(x^{\prime}, y^{\prime}\right) G^{m}\left(x, y, t \mid x^{\prime}, y^{\prime}, 0\right) d x^{\prime} d y^{\prime}
\end{aligned}
$$

for the periodic array of fractures problem.

In this analysis, we assumed that there is a source distribution at $y=0$, namely $S(x, t)$, along the whole fracture-matrix system. Another typical boundary condition is:

$$
\begin{gathered}
c(x, 0, t)=S(x, t) \text { in the fracture } \\
\theta^{m}\left[-D_{22}^{m} \frac{\partial c}{\partial y}(x, 0, t)+V^{m} c(x, 0, t)\right]=F(x, t) \text { in the matrix }
\end{gathered}
$$

where $F(x, t)$ is the mass flux per unit area of matrix across the top of the porous matrix. Clearly the fracture Green's function, $G^{f}$, and the representation for the fracture concentration, (20), remain the same if condition (26) applies, with $I^{f}$ defined in (21). When the concentration is subject to a known mass flux $F(x, t)$ instead of a source distribution, the boundary condition on $G^{m}$ at $y^{\prime}=0$ must be modified in order to obtain a representation of the matrix concentration in terms of known functions on the boundary. Instead of conditions (22a) and (23a) we require for both geometries

$$
\frac{\partial G^{m}}{\partial y^{\prime}}\left(x, y, t \mid x^{\prime}, 0, t^{\prime}\right)=0
$$

on all the $x^{\prime}$ matrix domain and $t^{\prime}>0$. Using a generalized notation, the representation for the matrix concentration, (24), remains valid, but the $G^{m}$ and $I^{m}$ will be different for both geometries. It can be easily verified that

$$
\begin{aligned}
I^{m}(x, y, t)= & -\frac{1}{\theta^{m}} \int_{0}^{t} \int_{b}^{\infty} F\left(x^{\prime}, t^{\prime}\right) G^{m}\left(x, y, t \mid x^{\prime}, 0, t^{\prime}\right) d x^{\prime} d t^{\prime} \\
& -R^{m} \int_{0}^{\infty} \int_{b}^{\infty} c_{0}\left(x^{\prime}, y^{\prime}\right) G^{m}\left(x, y, t \mid x^{\prime}, y^{\prime}, 0\right) d x^{\prime} d y^{\prime}
\end{aligned}
$$

for the single fracture problem and

$$
\begin{aligned}
I^{m}(x, y, t)= & -\frac{1}{\theta^{m}} \int_{0}^{t} \int_{b}^{B} F\left(x^{\prime}, t^{\prime}\right) G^{m}\left(x, y, t \mid x^{\prime}, 0, t^{\prime}\right) d x^{\prime} d t^{\prime} \\
& -R^{m} \int_{0}^{\infty} \int_{b}^{B} c_{0}\left(x^{\prime}, y^{\prime}\right) G^{m}\left(x, y, t \mid x^{\prime}, y^{\prime}, 0\right) d x^{\prime} d y^{\prime}
\end{aligned}
$$

for the parallel array of fractures problem. The Green's functions $G^{m}$ will be given in the next section.

We return now to a unified discussion of the concentration representations in the fracture and matrix regions, given in (20) and (24). It must be emphasised 
that we are employing a generalised notation here. The explicit forms of $G^{f}$, $G^{m}, I^{f}$ and $I^{m}$ are dependent upon which of the two geometries and which of the two types of concentration boundary conditions at the top of the matrix region is being considered.

Let the mass flux in the matrix, at the fracture-matrix interface, be denoted by

$$
J(y, t)=\theta^{m} D_{11}^{m} \frac{\partial c}{\partial x}\left(b^{+}, y, t\right)
$$

Then applying the interfacial conditions (18a) and (18b) to (20) and (24) gives expressions for the concentration everywhere in the system in terms of $J$ :

$$
c(x, y, t)=-\frac{1}{\theta^{f}} \int_{0}^{t} \int_{0}^{\infty} G^{f}\left(x, y, t \mid b^{-}, y^{\prime}, t^{\prime}\right) J\left(y^{\prime}, t^{\prime}\right) d y^{\prime} d t^{\prime}+I^{f}(x, y, t)
$$

in the fracture and

$$
c(x, y, t)=\frac{1}{\theta^{m}} \int_{0}^{t} \int_{0}^{\infty} G^{m}\left(x, y, t \mid b^{+}, y^{\prime}, t^{\prime}\right) J\left(y^{\prime}, t^{\prime}\right) d y^{\prime} d t^{\prime}+I^{m}(x, y, t)
$$

in the matrix, and an integral equation of the first kind to be solved for $J$ :

$$
\begin{aligned}
\int_{0}^{t} \int_{0}^{\infty} & \left(\frac{1}{\theta^{f}} G^{f}\left(b^{-}, y, t \mid b^{-}, y^{\prime}, t^{\prime}\right)+\frac{1}{\theta^{m}} G^{m}\left(b^{+}, y, t \mid b^{+}, y^{\prime}, t^{\prime}\right)\right) J\left(y^{\prime}, t^{\prime}\right) d y^{\prime} d t^{\prime} \\
& =I^{f}\left(b^{-}, y, t\right)-I^{m}\left(b^{+}, y, t\right) .
\end{aligned}
$$

\section{Fracture and matrix Green's functions}

The fracture Green's function is found by making the transformation

$$
G^{f}\left(x, y, t \mid x^{\prime}, y^{\prime}, t^{\prime}\right)=\frac{-1}{2 \pi} \exp \left(\frac{-V^{f}}{2 D_{22}^{f}}\left(y^{\prime}-y\right)\right) H^{f}\left(x, y, t \mid x^{\prime}, y^{\prime}, t^{\prime}\right)
$$

and then solving the self-adjoint equation for $H^{f}$ using standard Fourier series and transform techniques. It can be written as

$$
\begin{aligned}
H^{f}\left(x, y, t \mid x^{\prime}, y^{\prime}, t^{\prime}\right)= & \left(h_{1}^{f}\left(\left|x_{1}-x_{2}\right|, y_{1}, t-t^{\prime}\right)+h_{1}^{f}\left(x_{1}+x_{2}, y_{1}, t-t^{\prime}\right)\right) \\
& -\left(h_{1}^{f}\left(\left|x_{1}-x_{2}\right|, y_{2}, t-t^{\prime}\right)+h_{1}^{f}\left(x_{1}+x_{2}, y_{2}, t-t^{\prime}\right)\right)
\end{aligned}
$$

where the function $h_{1}^{f}$ is defined as

$$
h_{1}^{f}(X, Y, T)=\frac{1}{\sqrt{D_{22}^{f} R^{f} \pi}} \frac{1}{l^{f}} \frac{1}{\sqrt{T}} \exp \left(\frac{-R^{f}}{4 D_{22}^{f}} \frac{Y^{2}}{T}\right) \sum_{n=0}^{\infty} \exp \left(-p_{n}^{f} T\right) \cos \left(\frac{n X}{l^{f}}\right) .
$$


Here the prime on the summation symbol signifies that the $n=0$ term is weighted by one half. The symbols introduced in the above two equations are defined as

$$
\begin{gathered}
x_{1}=\left|x^{\prime}-b\right|, \quad x_{2}=|x-b| \\
y_{1}=\left|y^{\prime}-y\right|, \quad y_{2}=y^{\prime}+y \\
l^{f}=\frac{b}{\pi} \\
p_{n}^{f}=\frac{1}{R^{f}}\left(\lambda R^{f}+\frac{1}{D_{22}^{f}}\left(\frac{V^{f}}{2}\right)^{2}+D_{11}^{f}\left(\frac{n}{l^{f}}\right)^{2}\right) .
\end{gathered}
$$

The matrix Green's function for both geometries and boundary condition at $y^{\prime}=0$ is initiated by making the transformation

$$
G^{m}\left(x, y, t \mid x^{\prime}, y^{\prime}, t^{\prime}\right)=\frac{-1}{2 \pi} \exp \left(\frac{-V^{m}}{2 D_{22}^{m}}\left(y^{\prime}-y\right)\right) H^{m}\left(x, y, t \mid x^{\prime}, y^{\prime}, t^{\prime}\right)
$$

and then solving the self-adjoint equation for $H^{m}$ using standard techniques. The solutions for both geometries and boundary conditions are given in Table 1 in terms of the following functions

$$
\begin{aligned}
& h_{1}^{m}(X, Y, T)= \frac{1}{\sqrt{D_{22}^{m} R^{m} \pi}} \frac{1}{l^{m}} \frac{1}{\sqrt{T}} \exp \left(\frac{-R^{m}}{4 D_{22}^{m}} \frac{Y^{2}}{T}\right) \sum_{n=0}^{\infty} \exp \left(-p_{n}^{m} T\right) \cos \left(\frac{n X}{l^{m}}\right) \\
& h_{2}^{m}(X, Y, T)= \frac{1}{2 \sqrt{D_{11}^{m} D_{22}^{m}}} \frac{1}{T} \exp \left(-p_{0}^{m} T\right) \exp \left(\frac{-R^{m}}{4 T}\left(\frac{Y^{2}}{D_{22}^{m}}+\frac{X^{2}}{D_{11}^{m}}\right)\right) \quad(38 \mathrm{a}) \\
& h_{3}^{m}(X, Y, T)= \frac{V^{m}}{D_{22}^{m} R^{m}} \frac{1}{l^{m}} \exp \left(\frac{V^{m}}{2 D_{22}^{m}} Y\right) \exp \left(\left(\frac{V^{m}}{2}\right)^{2} \frac{T}{D_{22}^{m} R}\right) \\
& \times \operatorname{erfc}\left(\frac{1}{2} \sqrt{\left.\frac{R^{m}}{D_{22}^{m} T} Y+\frac{V^{m}}{2} \sqrt{\frac{T}{D_{22}^{m} R^{m}}}\right) \sum_{n=0}^{\infty} \exp \left(-p_{n}^{m} T\right) \cos \left(\frac{n X}{l^{m}}\right)}\right. \\
& h_{4}^{m}(X, Y, T)= \frac{V^{m}}{2 D_{22}^{m}} \sqrt{\frac{\pi}{D_{11}^{m} R^{m}} \frac{1}{\sqrt{T}}} \exp \left(\frac{V^{m}}{2 D_{22}^{m}} Y-\frac{R^{m}}{4 D_{11}^{m}} \frac{X^{2}}{T}-\lambda T\right) \\
& \times \operatorname{erfc}\left(\frac{1}{2} \sqrt{\frac{R^{m}}{D_{22}^{m} T}} Y+\frac{V^{m}}{2} \sqrt{\frac{T}{D_{22}^{m} R^{m}}}\right)
\end{aligned}
$$

The symbols introduced here are

$$
\begin{gathered}
l^{m}=(B-b) / \pi \\
p_{n}^{m}=\frac{1}{R^{m}}\left(\lambda R^{m}+\frac{1}{D_{22}^{m}}\left(\frac{V^{m}}{2}\right)^{2}+D_{11}^{m}\left(\frac{n}{l^{m}}\right)^{2}\right) .
\end{gathered}
$$

Note that the expressions for $h_{1}^{m}$ and $h_{3}^{m}$ can be viewed as discretisations of the Fourier cosine transform integrals defining the functions $h_{2}^{m}$ and $h_{4}^{m}$ in which the 


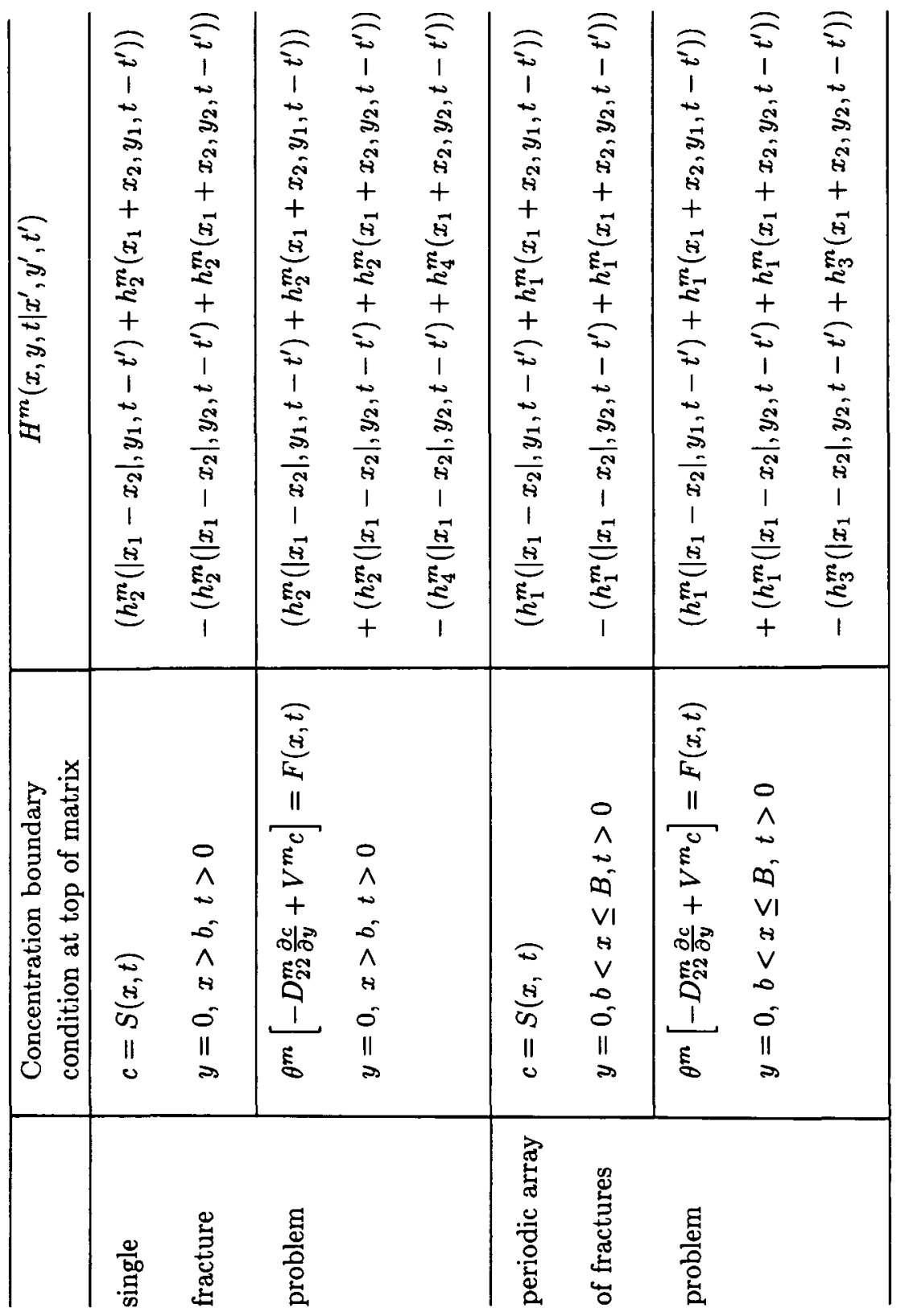


integral is replaced by an infinite sum of rectangle areas. Further, these discretisations converge to the exact integrals as $B-b \rightarrow \infty$. So the Green's function for the periodic array of fractures problem reproduces the Green's function of the single fracture problem in the limit of the intervening porous block width becoming infinite, as expected. It should also be noted that if the functions in the boundary conditions, namely $S$ and $F$ are independent of time, the integral representations of $c(x, y, t)$ in the limit as $t \rightarrow \infty$, that is the steady state concentration, reduce to those in [3].

\section{Integral equation}

In the fracture-matrix systems above we have shown that the concentration in each region can be written in terms of an integral of the interfacial mass flux $J$ and that this flux is the solution to an integral equation of the first kind. In fact it follows from the discussion in Section 4 that this is true for any two adjacent media problem. Hence the concentration of contaminant in a two media system is determined at any position at any time once an integral equation of the form

$$
\int_{0}^{t} \int_{\substack{\text { interface } \\ \text { boundary }}} K\left(y, y^{\prime}, t-t^{\prime}\right) J\left(y^{\prime}, t^{\prime}\right) d y^{\prime} d t^{\prime}=g(y, t)
$$

is solved for the interfacial flux $J$. Here $y^{\prime}$ is the variable which parametrises the interface boundary and the functions $K$ and $g$ are known. The kernel $K$ is a linear combination of the Green's functions in the two media and clearly its dependence on $t$ and $t^{\prime}$ is only through $t-t^{\prime}$. The function $g$ is composed of integrals dependent on the Green's functions and the concentration boundary and initial conditions. Analytical solution of this integral equation is not possible in general. We briefly outline an efficient and reliable numerical procedure for solving this equation. The discretisation procedure is analogous to the one described and implemented in our previous paper on the steady state problem [3].

If the interface boundary has infinite length then the infinite integration range in $y^{\prime}$ must be replaced by a finite range, say $[0, A]$ for some sufficiently large value of $\boldsymbol{A}$. Hence for both a finite and infinite length boundary, we consider the equation

$$
\int_{0}^{t} \int_{0}^{A} K\left(y, y^{\prime}, \tau\right) J\left(y^{\prime}, t-\tau\right) d y^{\prime} d \tau=g(y, t)
$$

where for convenience we have changed the $t^{\prime}$ integration variable to $\tau=t-t^{\prime}$. Now partition the finite $y^{\prime}$ integration range using the set of points $\left\{y_{j}\right\}_{j=1}^{N}$, where

$$
y_{1}=\Delta / 2 ; \quad y_{j+1}=y_{j}+\Delta, j=1,2, \ldots, N-1 ; \quad y_{N}+(\Delta / 2)=A
$$


(This can be easily extended to nonequal $\Delta_{j}$ 's, $j=1,2, \ldots, N$ ). Further let $t=n \delta, n=1,2, \ldots$. Then provided that the function $J(y, t)$ is sufficiently slowly varying in each subinterval of width $\Delta$ in $y$ and $\delta$ in $t$,

$$
\begin{aligned}
\int_{0}^{t} \int_{0}^{A} K\left(y, y^{\prime}, \tau\right) J\left(y^{\prime}, t-\tau\right) d y^{\prime} d \tau & \\
& \approx \sum_{r=1}^{n} \sum_{j=1}^{N} J\left(y_{j},(n-r+1 / 2) \delta\right) \int_{(r-1) \delta}^{r \delta} \int_{y_{j}-\Delta / 2}^{y_{j}+\Delta / 2} K\left(y, y^{\prime}, \tau\right) d y^{\prime} d \tau
\end{aligned}
$$

so that equation (41) has the approximate form

$$
\sum_{r=1}^{n} \sum_{j=1}^{N} J\left(y_{j},(n-r+1 / 2) \delta\right) \int_{(r-1) \delta}^{r \delta} \int_{y_{j}-\Delta / 2}^{y,+\Delta / 2} K\left(y, y^{\prime}, \tau\right) d y^{\prime} d \tau=g(y, n \delta)
$$

Choosing $y=y_{i}, i=1,2, \ldots, N$, in this equation produces for each value of $n$ a system of $N$ simultaneous linear equations for the unknown $J(y, t)$ values at each of the $N$ points of the interface boundary partition evaluated at times $(n-(1 / 2)) \delta$.

Using matrix notation we have

$$
\begin{gathered}
\mathbf{K}^{(1)} \mathbf{J}(\delta / 2)=\mathbf{g}(\delta) \\
\mathbf{K}^{(1)} \mathbf{J}((n-1 / 2) \delta)=\mathbf{g}(n \delta)-\sum_{r=2}^{n} \mathbf{K}^{(r)} \mathbf{J}((n-r+1 / 2) \delta) \quad n=2,3, \ldots
\end{gathered}
$$

where

$$
\begin{gathered}
\mathbf{K}^{(r)}=\left(K_{i j}^{(r)}\right), \quad K_{i j}^{(r)}=\int_{(r-1) \delta}^{r \delta} \int_{y_{j}-\Delta / 2}^{y_{j}+\Delta / 2} K\left(y_{i}, y^{\prime}, \tau\right) d y^{\prime} d \tau \\
\mathbf{J}(\alpha)=\left(J_{j}(\alpha)\right), \quad J_{j}(\alpha)=J\left(y_{j}, \alpha\right) \\
\mathbf{g}(\alpha)=\left(g_{i}(\alpha)\right), \quad g_{i}(\alpha)=g\left(y_{i}, \alpha\right) .
\end{gathered}
$$

Therefore, once $\mathbf{J}(\delta / 2)$ is calculated from (45a) the $\mathbf{J}(3 \delta / 2), \mathbf{J}(5 \delta / 2), \ldots$, $\mathrm{J}((n-1 / 2) \delta)$ can be obtained in succession using (45b). The simplicity of this time-stepping procedure lies in the fact that the matrix which multiplies the unknown $\mathbf{J}((n-1 / 2) \delta)$ is always $\mathbf{K}^{(1)}$. The entries of this matrix must be computed with sufficient accuracy to ensure reliable values of $\mathbf{J}$. For the fracture-matrix systems discussed in Sections 5 and 6 , the kernel function in (46a) defining $\mathbf{K}^{(1)}$ is singular at $\tau=0$. The $y^{\prime}$ and then the $\tau$ integration can be performed analytically with the help of tables [1]. In general, if analytical expressions for the integrals cannot be obtained, then the singular part of the kernel should be integrated analytically, while the remainder can be integrated using quadrature (as discussed and implemented in [3]). 
The integral representation for the concentration in each region can be discretised in a similar fashion using the partition $\left\{y_{j}\right\}$ along the interface boundary and $t=n \delta(n=1,2 \ldots)$.

\section{Conclusion}

A general method using boundary integral techniques has been proposed for the solution of contaminant transport by two-dimensional diffusion and advection into two adjacent porous media. The method reduces to solving an integral equation for the mass flux on the interfacial boundary between the two media. The method has been outlined in detail for certain fracture-matrix systems, but can easily be applied to other types of problems.

\section{References}

[1] M. Abramowitz and I. A. Stegun, Handbook of mathematical funtions. (Dover, New York, 1972).

[2] J. Bear, Dynamics of fluzds in porous medra. (Elsevier, New York, 1972).

[3] A. Fogden, K. A. Landman and L. R. White, "Contaminant transport in fractured porous media: steady state solutions by a boundary integral method", to appear in Water Resour. Res. (1988).

[4] A. Fogden, K. A. Landman and L. R. White, "Contaminant transport in fractured porous media: steady state solutions by Fourier sine transform method", submitted.

[5] G. E. Grisak and J. F. Pickens, "Solute transport through fractured media. I: The effect of matrix diffusion", Water Resour. Res. 16 (1980) 719-730.

[6] G. E. Grisak and J. F. Pickens, "An analytical solution for solute transport through fractured media with matrix diffusion", J. Hydrology 52 (1981) 47-57.

[7] P. S. Huyakorn, B. H. Lester and J. W. Mercer, "An efficient finite element technique for modelling transport in fractured porous media. I: Single species transport", Water Resour. Res. 19 (1983) 841-854.

[8] I. Neretnieks, "Diffusion in a rock matrix: an important factor in radionuclide retardation?" J. Geophys. Res. 85 (1980) 4379-4397.

[9] J. Noorishad and M. Mehran, "An upstream finite element method for solution of transient transport equation in fractured porous media", Water Resour. Res. 18 (1982) 588596.

[10] E. A. Sudicky and E. O. Frind, "Contaminant transport in a fractured porous media; analytical solutions for a system of parallel fractures", Water Resour. Res. 18 (1982) $1634-1642$.

[11] A. Taigbenu and J. A. Liggett, "An integral solution for the diffusion-advection equation", Water Resour. Res. 22 (1986) 1237-1246.

[12] D. H. Tang, E. O. Frind and E. A. Sudicky, "Contaminant transport in fractured porous media: analytical solution for a single fracture", Water Resour. Res. 17 (1981) 555-564. 